Annals of Pure and Applied Mathematics

Vol. 15, No. 1, 2017, 89-95

ISSN: 2279-087X (P), 2279-0888(online)

Published on 11 December 2017

www.researchmathsci.org

DOI: http://dx.doi.org/10.22457/apam.v15n1a8

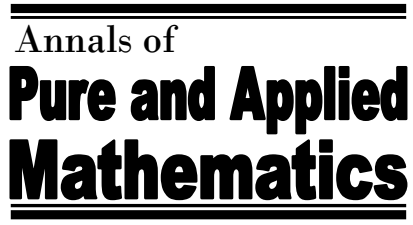

\title{
Connectivity in a Bipolar Fuzzy Graph and its Complement
}

\author{
S.Yahya Mohamed ${ }^{1}$ and N.Subashini ${ }^{2}$ \\ ${ }^{1}$ Department of Mathematics, Government Arts College \\ Tiruchirappalli-620022, India. E-mail: yahya md@yahoo.com \\ ${ }^{2}$ Department of Mathematics, Saranathan College of Engineering \\ Tiruchirappalli-620012, India. E-mail:yazhinisuba@gmail.com
}

Received 1 November 2017; accepted 4 December 2017

\begin{abstract}
Connectivity has important role in the area of applications of fuzzy graphs such as fuzzy neural networks and clustering. In this paper criterion for connectivity of a bipolar fuzzy graph and its complement is analyzed. The structure of the complement of a bipolar fuzzy cycle is also discussed.
\end{abstract}

Keywords: Fuzzy relations, complement of bipolar fuzzy graph, bipolar fuzzy cycle, connectivity in bipolar fuzzy graphs, m-strong arcs.

\section{AMS Mathematics Subject Classification (2010): $05 \mathrm{C} 72$}

\section{Introduction}

In 1965, Zadeh [1] initiated the concept of bipolar fuzzy sets as a generalization of fuzzy sets. Bipolar fuzzy sets are an extension of fuzzy sets whose membership degree range is $[-1,1]$. In a bipolar fuzzy set, the membership degree 0 of an element means that the element is irrelevant to the corresponding property, the membership degree $(0,1]$ of an element indicates that the element somewhat satisfies the property, and the membership degree $[-1,0)$ of an element indicates that the element somewhat satisfies the implicit counter-property. Although bipolar fuzzy sets and intuitionistic fuzzy sets look similar to each other, they are essentially different sets. In many domains, it is important to be able to deal with bipolar fuzzy information. It is noted that positive information represents what is granted to be possible, while negative information represents what is considered to be impossible. This domain has recently motivated new research in several directions. Akram[2] introduced the concept of bipolar fuzzy graphs and defined different operations on it. Narayanamoorthy and Tamilselvi [3] introduced the concept of bipolar fuzzy line graph of a bipolar fuzzy hypergraph. Mathew, Sunitha and Anjali [4] introduced the concept of complement bipolar fuzzy graphs in 2014 and discussed about some connectivity concepts in bipolar fuzzy graphs. Connectivity has important role in the area of applications of fuzzy graphs such as fuzzy neural networks and clustering. In this paper criterion for connectivity of a bipolar fuzzy graph and its complement is analyzed. The structure of the complement of a bipolar fuzzy cycle is also discussed. 


\section{S.Yahya Mohamed and N.Subashini}

\section{Preliminaries}

In this section, some basic definitions and preliminary ideas are given which is useful for proving theorems.

Definition 2.1. [1] By a bipolar fuzzy graph, we mean a pair $\mathrm{G}=(\mathrm{A}, \mathrm{B})$ where $A=$ $\left(\mu_{A}^{P}, \mu_{A}^{N}\right)$ is a bipolar fuzzy set in $\mathrm{V}$ and $B=\left(\mu_{B}^{P}, \mu_{B}^{N}\right)$ is a bipolar fuzzy relation on $\mathrm{E}$ such that $\mu_{B}^{P}(x y) \leq \min \left(\mu_{A}^{P}(x), \mu_{A}^{P}(y)\right)$ and $\mu_{B}^{N}(x y) \geq \max \left(\mu_{A}^{N}(x), \mu_{A}^{N}(y)\right)$ for $\operatorname{all}(x, y) \in E$.

Definition 2.2. [1] Let A be the bipolar fuzzy vertex set of $\mathrm{V}, \mathrm{B}$ the bipolar fuzzy edge set of $E$ respectively. Note that $B$ is symmetric bipolar fuzzy relation on A. we use the notation xy for an element of $\mathrm{E}$. Thus, $\mathrm{G}=(\mathrm{A}, \mathrm{B})$ is a bipolar fuzzy graph of $G^{*}=(V, E)$ if

$$
\mu_{B}^{P}(x y) \leq \min \left(\mu_{A}^{P}(x), \mu_{A}^{P}(y)\right) \text { and } \mu_{B}^{N}(x y) \geq \max \left(\mu_{A}^{N}(x), \mu_{A}^{N}(y)\right) \text { for all } x y \in
$$

$E$. $H=(\alpha, \beta)$ (where $\alpha=\left(\mu_{A}^{P}, \mu_{A}^{N}\right)$ is a bipolar fuzzy subset of a set A and $\beta=\left(\mu_{B}^{P}, \mu_{B}^{N}\right)$ is a bipolar fuzzy relation on $\mathrm{B}$ ) is called a partial bipolar fuzzy subgraph of $\mathrm{G}$ if $\alpha \leq A$ and $\beta \leq B$. We call $H=(\alpha, \beta)$ a spanning bipolar fuzzy subgraph of $G=(A, B)$ if $\alpha=A$.

Definition 2.3. [2] A path $\mathrm{p}$ of length $\mathrm{n}$ is a sequence of distinct nodes

$\left(\left(\mu_{A}^{p}\left(u_{0}\right), \mu_{A}^{N}\left(u_{0}\right)\right)\left(\mu_{A}^{p}\left(u_{1}\right), \mu_{A}^{N}\left(u_{1}\right)\right)\left(\mu_{A}^{p}\left(u_{2}\right), \mu_{A}^{N}\left(u_{2}\right)\right) \ldots .\left(\mu_{A}^{p}\left(u_{n}\right), \mu_{A}^{N}\left(u_{n}\right)\right)\right)$ such that $\left(\mu_{B}^{P}\left(u_{i-1} u_{i}\right), \mu_{B}^{N}\left(u_{i-1} u_{i}\right)\right)>0$ and degree of membership of a weakest arc is defined as its strength. If $\left(\mu_{A}^{p}\left(u_{0}\right), \mu_{A}^{N}\left(u_{0}\right)\right)=\left(\mu_{A}^{p}\left(u_{n}\right), \mu_{A}^{N}\left(u_{n}\right)\right)$ and $n \geq 3$, then $\mathrm{p}$ is called a cycle and it is a bipolar fuzzy cycle if there is more than one weak arc.

Definition 2.4. [3] The strength of connectedness between 2 nodes $\left(\mu_{A}^{p}(x), \mu_{A}^{N}(x)\right),\left(\mu_{A}^{p}(y), \mu_{A}^{N}(y)\right)$ is defined as the maximum of strengths of all paths between $\mathrm{x}$ and $\mathrm{y}$ and is denoted byCONN $\mathrm{CON}_{G}(x, y)$.

Definition 2.5. [3] An $\operatorname{arc}\left(\left(\mu_{A}^{p}(x), \mu_{A}^{N}(x)\right),\left(\mu_{A}^{p}(y), \mu_{A}^{N}(y)\right)\right)$ is called a fuzzy bridge in $\mathrm{G}$ if the removal of $\left(\left(\mu_{A}^{p}(x), \mu_{A}^{N}(x)\right),\left(\mu_{A}^{p}(y), \mu_{A}^{N}(y)\right)\right)$ reduces the strength of connectedness between some pair of nodes in G. A connected bipolar fuzzy graph is called a bipolar fuzzy tree if it contains a spanning subgraph $\mathrm{F}$ which is a tree such that for all $\operatorname{arcs}\left(\left(\mu_{A}^{p}(x), \mu_{A}^{N}(x)\right),\left(\mu_{A}^{p}(y), \mu_{A}^{N}(y)\right)\right)$ not in F,

$$
\left(\mu_{B}^{P}(x y), \mu_{B}^{N}(x y)\right)<\operatorname{CONN}_{F}\left(\left(\mu_{A}^{p}(x), \mu_{A}^{N}(x)\right),\left(\mu_{A}^{p}(y), \mu_{A}^{N}(y)\right)\right) .
$$

Definition 2.6. [4] An $\operatorname{arc}\left(\left(\mu_{A}^{p}(u), \mu_{A}^{N}(u)\right),\left(\mu_{A}^{p}(v), \mu_{A}^{N}(v)\right)\right)$ of $\mathrm{G}$ is called m-strong if $\mu_{B}^{P}(u, v)=\min \left(\mu_{A}^{P}(u), \mu_{A}^{P}(v)\right)$ and $\mu_{B}^{N}(u, v)=\max \left(\mu_{A}^{N}(u), \mu_{A}^{N}(v)\right)$. Suppose $\mathrm{G}:(\mathrm{A}, \mathrm{B})$ be a bipolar fuzzy graph. The complement of $\mathrm{G}$ is denoted as $\bar{G}:(\bar{A}, \bar{B})$ where $\bar{A}=A$ and $\bar{\mu}_{B}^{P}(x y)=\min \left(\mu_{A}^{P}(x), \mu_{A}^{P}(y)\right)-\mu_{B}^{P}(x y) \&$ 
Connectivity in a Bipolar Fuzzy Graph and its Complement

$\bar{\mu}_{B}^{N}(x y)=\max \left(\mu_{A}^{N}(x), \mu_{A}^{N}(y)\right)-\mu_{B}^{N}(x y)$.

In all the examples of this paper we assume that the membership value of each node is $[-1,1]$ unless otherwise specified.

\section{Connectivity in $G^{c}$}

In this section, we propose a criterion by which a bipolar fuzzy graph and its complement will be connected simultaneously.

Example 3.1. A bipolar fuzzy graph on 3 vertices and its complement
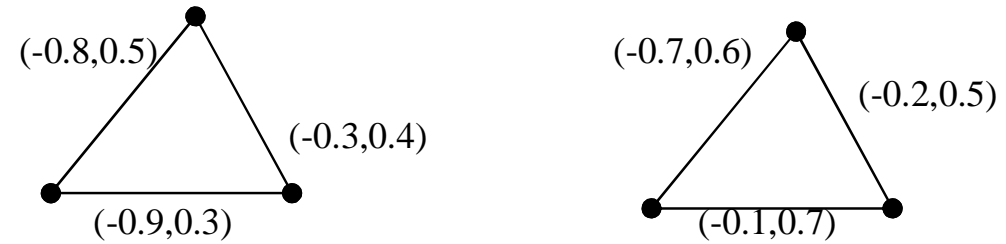

Figure 1: Bipolar fuzzy graph and its complement

Proposition 3.2. Let $G=(A, B)$ be a connected bipolar fuzzy graph with no $\mathrm{m}$-strong arcs then $G^{c}$ is connected.

Proof: The bipolar fuzzy graph $\mathrm{G}$ is connected and contain no $\mathrm{m}$-strong arcs. Suppose $\left(\mu_{A}^{p}(u), \mu_{A}^{N}(u)\right),\left(\mu_{A}^{p}(v), \mu_{A}^{N}(v)\right)$ be two arbitrary nodes of $G^{c}$. Then they are also nodes of G. Since G is connected there exist a path between $\left(\mu_{A}^{p}(u), \mu_{A}^{N}(u)\right) \&\left(\mu_{A}^{p}(v), \mu_{A}^{N}(v)\right)$ in G. Let this path be P.

Then

$$
\begin{gathered}
\mathrm{P}=\left[\left(\mu_{A}^{p}\left(u_{0}\right), \mu_{A}^{N}\left(u_{0}\right)\right)\left(\mu_{A}^{p}\left(u_{1}\right), \mu_{A}^{N}\left(u_{1}\right)\right)\right],\left[\left(\mu_{A}^{p}\left(u_{1}\right), \mu_{A}^{N}\left(u_{1}\right)\right)\left(\mu_{A}^{p}\left(u_{2}\right), \mu_{A}^{N}\left(u_{2}\right)\right)\right] \ldots \\
{\left[\left(\mu_{A}^{p}\left(u_{n-1}\right), \mu_{A}^{N}\left(u_{n-1}\right)\right)\left(\mu_{A}^{p}\left(u_{n}\right), \mu_{A}^{N}\left(u_{n}\right)\right)\right]}
\end{gathered}
$$

where $\left(\mu_{B}^{P}\left(u_{i-1} u_{i}\right), \mu_{B}^{N}\left(u_{i-1} u_{i}\right)\right)>0$.

Since $\mathrm{G}$ contains no $\mathrm{m}$-strong arcs, $\left(\mu_{B^{c}}^{P}\left(u_{i-1} u_{i}\right), \mu_{B^{c}}^{N}\left(u_{i-1} u_{i}\right)\right)>0$ for every $i$

Hence $\mathrm{P}$ will be a $\left(\mu_{A}^{p}(u), \mu_{A}^{N}(u)\right),\left(\mu_{A}^{p}(v), \mu_{A}^{N}(v)\right)$ path in $G^{c}$ also. Therefore $G^{c}$ is connected.

Remark 3.3. There are bipolar fuzzy graphs which contain $m$-strong arcs such that $G$ and $G^{c}$ are connected (Figure 2)
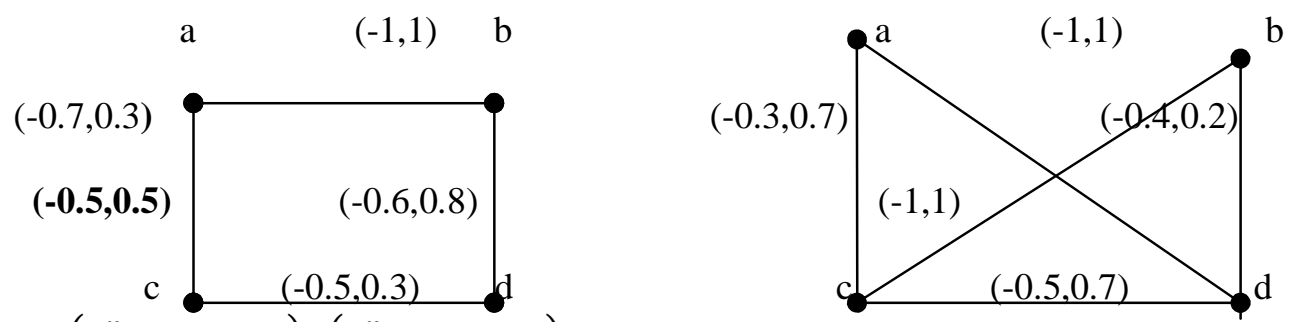

Figure 2: $\left(\mu_{A}^{p}(a), \mu_{A}^{N}(a)\right),\left(\mu_{A}^{p}(b), \mu_{A}^{N}(b)\right)$ is an m-strong arc in $\mathrm{G}$ and $G^{c}$ is connected 


\section{S.Yahya Mohamed and N.Subashini}

Theorem 3.4. Let $G=(A, B)$ be a bipolar fuzzy graph. $\mathrm{G}$ and $G^{c}$ are connected if and only if $\mathrm{G}$ contains atleast one connected spanning bipolar fuzzy subgraph with no $\mathrm{m}$ strong arcs.

Proof: Suppose that $\mathrm{G}$ contains a spanning bipolar fuzzy subgraph $\mathrm{H}$ that is connected, having no m- strong arcs. Since $\mathrm{H}$ contains no $\mathrm{m}$-strong arcs and is connected using proposition-1, $H^{c}$ will be a connected spanning bipolar fuzzy subgraph of $G^{c}$ and thus $G^{c}$ is also connected.

Conversely assume that $\mathrm{G}$ and $G^{c}$ are connected. We have to find a connected spanning bipolar fuzzy subgraph of $\mathrm{G}$ that contain no $\mathrm{m}$-strong arcs.

Let $\mathrm{H}$ be an arbitrary connected spanning bipolar fuzzy subgraph of $\mathrm{G}$. If $\mathrm{H}$ contain no $\mathrm{m}$-strong arcs then $\mathrm{H}$ is required bipolar fuzzy subgraph. Suppose $\mathrm{H}$ contain one m-strong arc say $\left(\mu_{A}^{p}(u), \mu_{A}^{N}(u)\right),\left(\mu_{A}^{p}(v), \mu_{A}^{N}(v)\right)$. Then arc

$$
\left(\mu_{A}^{p}(u), \mu_{A}^{N}(u)\right),\left(\mu_{A}^{p}(v), \mu_{A}^{N}(v)\right) \text { will not be present in } G^{c} \text {. Since } G^{c} \text { is }
$$

connected there will exist a $\left(\mu_{A}^{p}(u), \mu_{A}^{N}(u)\right)-\left(\mu_{A}^{p}(v), \mu_{A}^{N}(v)\right)$ path in $G^{c}$. Let this path be $P_{1}$.

Let

$$
\begin{aligned}
& P_{1}=\left[\left(\mu_{A}^{p}\left(u_{1}\right), \mu_{A}^{N}\left(u_{1}\right)\right)\left(\mu_{A}^{p}\left(u_{2}\right), \mu_{A}^{N}\left(u_{2}\right)\right)\right],\left[\left(\mu_{A}^{p}\left(u_{2}\right), \mu_{A}^{N}\left(u_{2}\right)\right)\left(\mu_{A}^{p}\left(u_{3}\right), \mu_{A}^{N}\left(u_{3}\right)\right)\right] \ldots \\
& \qquad\left[\left(\mu_{A}^{p}\left(u_{n-1}\right), \mu_{A}^{N}\left(u_{n-1}\right)\right)\left(\mu_{A}^{p}\left(u_{n}\right), \mu_{A}^{N}\left(u_{n}\right)\right)\right] \\
& \text { where }\left[\left(\mu_{A}^{p}\left(u_{1}\right), \mu_{A}^{N}\left(u_{1}\right)\right)=\left(\mu_{A}^{p}(u), \mu_{A}^{N}(u)\right)\right] \&\left[\left(\mu_{A}^{p}\left(u_{n}\right), \mu_{A}^{N}\left(u_{n}\right)\right)=\left(\mu_{A}^{p}(v), \mu_{A}^{N}(v)\right)\right]
\end{aligned}
$$

If all the arcs of $P_{1}$ are present in $\mathrm{G}$ then $H-\left(\mu_{B}^{P}(u v), \mu_{B}^{N}(u v)\right)$ together with $P_{1}$ will be the required bipolar fuzzy spanning subgraph. If not, there exist atleast one arc say $\left[\left(\mu_{A}^{p}\left(u_{1}\right), \mu_{A}^{N}\left(u_{1}\right)\right)\left(\mu_{A}^{p}\left(v_{1}\right), \mu_{A}^{N}\left(v_{1}\right)\right)\right]$ in $P_{1}$ which is not in G. since G is connected we can replace

$\left[\left(\mu_{A}^{p}\left(u_{1}\right), \mu_{A}^{N}\left(u_{1}\right)\right),\left(\mu_{A}^{p}\left(v_{1}\right), \mu_{A}^{N}\left(v_{1}\right)\right)\right]$ by another

$\left[\left(\mu_{A}^{p}\left(u_{1}\right), \mu_{A}^{N}\left(u_{1}\right)\right)-\left(\mu_{A}^{p}\left(v_{1}\right), \mu_{A}^{N}\left(v_{1}\right)\right)\right]$

path in G. Let this path be $P_{2}$. If $P_{2}$ contain no $\mathrm{m}$-strong arcs then $H-\left[\left(\mu_{B}^{p}(u v), \mu_{B}^{N}(u v)\right)-\left(\mu_{B}^{p}\left(u_{1} v_{1}\right), \mu_{B}^{N}\left(u_{1} v_{1}\right)\right)\right]$ together with $P_{1}$ and $P_{2}$ will be the required spanning bipolar fuzzy subgraph. If $P_{2}$ contain an m-strong arc then this arc will not be present in $G^{c}$. Then replace this arc by a path connecting the corresponding vertices in $G^{c}$ and proceed as above and since $\mathrm{G}$ contain only finite number of arcs finally we will get a spanning bipolar fuzzy subgraph of that contain no m-strong arcs.

If more than one $\mathrm{m}$-strong arc is present in $\mathrm{H}$, then the above procedure can be repeated for all other $\mathrm{m}$-strong arcs of $\mathrm{H}$ to get the required spanning bipolar fuzzy subgraph of $\mathrm{G}$.

Corollary 3.5. Let $G=(A, B)$ be a bipolar fuzzy graph. $G$ and $G^{c}$ are connected if and only if $\mathrm{G}$ contains atleast one spanning bipolar fuzzy tree having no $\mathrm{m}$-strong arcs.

Proof: Using previous theorem we will get a connected spanning bipolar fuzzy subgraph of $\mathrm{G}$ which contain no $\mathrm{m}$-strong arcs. The maximum spanning bipolar fuzzy tree of this subgraph will be a spanning bipolar fuzzy tree of of $\mathrm{G}$ that contain no m-strong arcs. 


\section{Connectivity in a Bipolar Fuzzy Graph and its Complement}

\section{Complement of bipolar fuzzy cycles}

Next we examine the case of the complement of bipolar fuzzy cycles. By choosing the membership values of arcs and nodes suitably we can construct the complement of bipolar fuzzy cycles on 3, 4, 5 vertices as bipolar fuzzy cycles.

Example 4.1. $\mathrm{N}=3, \mathrm{G}$ and $G^{c}$ are both bipolar fuzzy cycles. (figure 3)
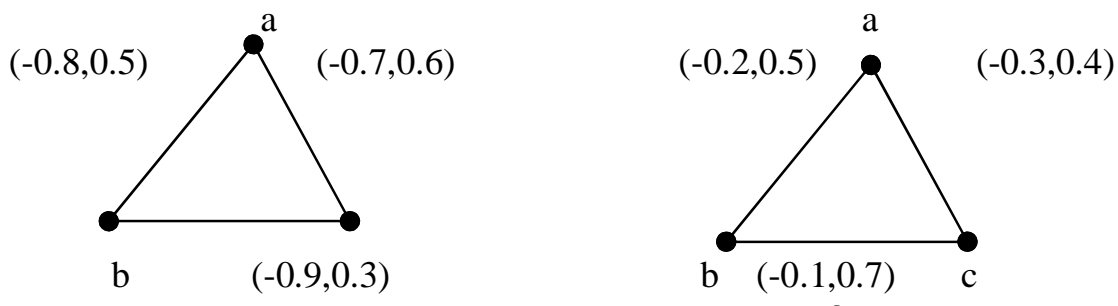

Figure 3: $c_{3}, c_{3}{ }^{c}$

Example 4.2. $\mathrm{N}=4, \mathrm{G}$ and $G^{c}$ are both bipolar fuzzy cycles. (figure 4)
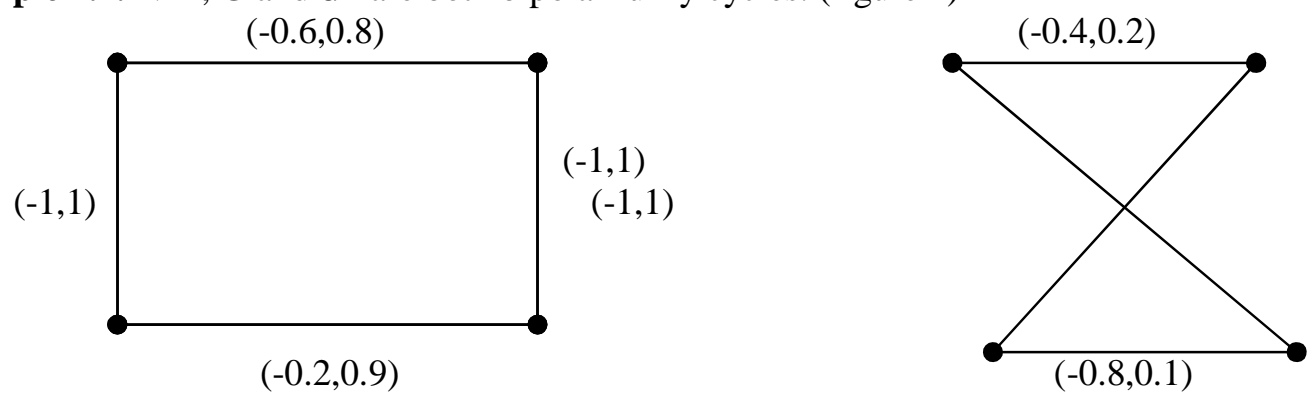

Figure 4: $c_{4}, c_{4}{ }^{c}$

Example 4.3. $\mathrm{N}=5, \mathrm{G}$ and $G^{c}$ are both bipolar fuzzy cycles. (figure 5)

\section{$(-1,1)$}

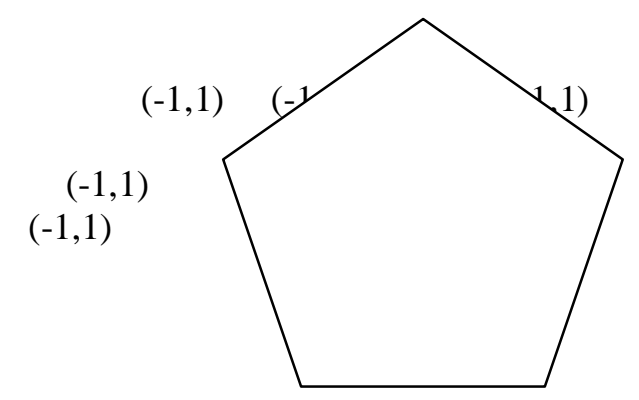

Figure 5: $c_{5}, c_{5}{ }^{c}$

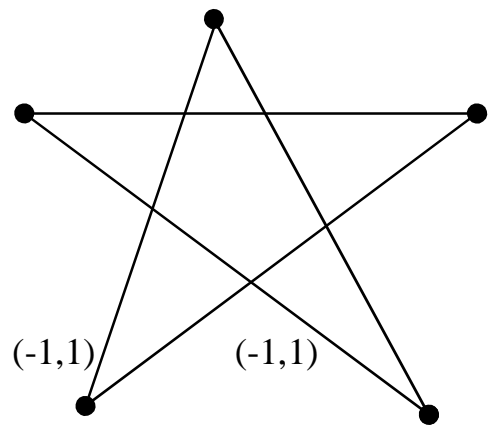

Theorem 4.4. Let $G:(A, B)$ be a bipolar fuzzy graph such that $G^{*}$ is a cycle with more than 5 vertices Then $\left(G^{*}\right)^{C}$ cannot be a cycle. 
S.Yahya Mohamed and N.Subashini

Proof: Given $G^{*}$ is a cycle having $\mathrm{n}$ nodes where $n \geq 6$. Then $G^{*}$ will have exactly $\mathrm{n}$ arcs. Since all the nodes of $\mathrm{G}$ are also present in $G^{c}$ number of nodes of $G^{c}$ is n. Let the nodes of $\mathrm{G}$ and $G^{c}$ be

$\left(\left(\mu_{A}^{p}\left(v_{1}\right), \mu_{A}^{N}\left(v_{1}\right)\right),\left(\mu_{A}^{p}\left(v_{2}\right), \mu_{A}^{N}\left(v_{2}\right)\right),\left(\mu_{A}^{p}\left(v_{3}\right), \mu_{A}^{N}\left(v_{3}\right)\right), \ldots .\left(\mu_{A}^{p}\left(v_{n}\right), \mu_{A}^{N}\left(v_{n}\right)\right)\right)$.

Then $G^{c}$ must contain atleast the following

edges. $\left\{\left(\mu_{B}^{P}\left(v_{1} v_{3}\right), \mu_{B}^{N}\left(v_{1} v_{3}\right)\right),\left(\mu_{B}^{P}\left(v_{1} v_{4}\right), \mu_{B}^{N}\left(v_{1} v_{4}\right)\right), \ldots .\left(\mu_{B}^{P}\left(v_{1} v_{n}\right), \mu_{B}^{N}\left(v_{1} v_{n}\right)\right)\right\}$;

$\left\{\left(\mu_{B}^{P}\left(v_{2} v_{4}\right), \mu_{B}^{N}\left(v_{2} v_{4}\right)\right),\left(\mu_{B}^{P}\left(v_{2} v_{5}\right), \mu_{B}^{N}\left(v_{2} v_{5}\right)\right), \ldots\left(\mu_{B}^{P}\left(v_{2} v_{n}\right), \mu_{B}^{N}\left(v_{2} v_{n}\right)\right)\right\} ;$

$\left\{\left(\mu_{B}^{P}\left(v_{3} v_{6}\right), \mu_{B}^{N}\left(v_{3} v_{6}\right)\right),\left(\mu_{B}^{P}\left(v_{3} v_{7}\right), \mu_{B}^{N}\left(v_{3} v_{7}\right)\right), \ldots\left(\mu_{B}^{P}\left(v_{3} v_{n}\right), \mu_{B}^{N}\left(v_{3} v_{n}\right)\right)\right\}$.

Since $n \geq 6$ the total number of edges in $G^{c}$ will be greater than n. Thus $G^{c}$ will not be a cycle.

Corollary 4.5. Let $\mathrm{G}$ be a bipolar fuzzy cycle with 6 or more nodes. Then $G^{c}$ will not be a bipolar fuzzy cycle.

\section{Conclusion}

In this paper, we have found some criterion for connectivity of a bipolar fuzzy graph and its complement and also studied some structure of the complement of a bipolar fuzzy cycle. The results discussed may be useful for further research in domination properties of bipolar fuzzy graphs.

\section{REFERENCES}

1. J.N.Mordeson and P.S.Nair, Fuzzy graphs and Fuzzy Hypergraphs, Physica Verlag (2000).

2. K.R.Bhutani and A.Rosefeld, Fuzzy end nodes in fuzzy graphs, Information Sciences, 152 (2003) 319-322.

3. L.A.Zadeh, Fuzzy sets, Information and Control, 8 (1965) 338-353.

4. M.Akram, Bipolar fuzzy graphs, Information Sciences, 181 (24) (2011) 5548-5564.

5. M.S.Sunitha and A.Vijayakumar, Complement of a fuzzy graph, Indian Journal of Pure and Applied Mathematics, 9(33) (2002) 1451-1464.

6. [6] M.S.Sunitha and A.Vijayakumar, Blocks in fuzzy graphs, Indian Journal of Fuzzy Mathematics, 13(1) (2005) 13-23.

7. M.S.Sunitha and A.Vijayakumar, A charecterisation of fuzzy trees, Information Sciences, 113 (2003) 319-322.

8. S.Narayanamoorthy and A.Tamilselvi, Bipolar fuzzy line graph of a bipolar fuzzy hypergraph, Cybernetics and Information Technologies, 13(1) (2013) 13-17.

9. S.Mathew, M.S.Sunitha and N.Anjali, Some connectivity in bipolar fuzzy graphs, Annals of Pure and Applied Mathematics, 7(2) (2014) 98-108.

10. S.Samanta and M.Pal, Irregular bipolar fuzzy graphs, International Journal of Applications of Fuzzy Sets, 2 (2012) 91-102.

11. S.Samanta and M.Pal, Bipolar fuzzy hypergraphs, International Journal of Fuzzy Logic Systems, 2(1) (2012) 17-28.

12. H.Rashmanlou and M.Pal, Some properties of highly irregular interval valued fuzzy graphs, World Applied Sciences Journal, 27(12) (2013) 1756-1773. 


\section{Connectivity in a Bipolar Fuzzy Graph and its Complement}

13. S.Samanta and M.Pal, Some more results on bipolar fuzzy sets and bipolar fuzzy intersection graphs, The Journal of Fuzzy Mathematics, 22(2) (2014) 253-262.

14. H.Rashmanlou, S.Samanta, M.Pal, R.A.Borzooei, A study on bipolar fuzzy graphs, Journal of Intelligent and Fuzzy Systems, 28 (2015) 571-580.

15. H.Rashmanlou, S.Samanta, M.Pal and R.A.Borzooei, Bipolar fuzzy graphs with Categorical properties, The International Journal of Computational Intelligence Systems, 8(5) (2015) 808-818.

16. H.Rashmanlou, S.Samanta, M.Pal, R.A.Borzooei, Product of bipolar fuzzy graphs and their degree, International Journal of General Systems, 45(1) (2016) 1-14.

17. G.Ghorai and M.Pal, Certain types of product bipolar fuzzy graphs, Intern. J. Applied and Computational Mathematics, 3(2) (2017) 605-619. 